\title{
Gallium nitride microcavities formed by photoenhanced wet oxidation
}

\author{
L.-H. Peng, ${ }^{\text {a) }}$ C.-Y. Lu, W.-H. Wu, and S.-L. Wang \\ Department of Electrical Engineering and Institute of Electro-Optical Engineering, \\ National Taiwan University, Taipei, Taiwan, Republic of China
}

(Received 22 June 2005; accepted 23 August 2005; published online 10 October 2005)

\begin{abstract}
We report the formation of gallium nitride $(\mathrm{GaN})$ microcavities by manipulating a photoenhanced oxidation rate difference between the polar and nonpolar crystallographic planes of GaN. When immersed in a buffered acetic $\left(\mathrm{CH}_{3} \mathrm{COOH}\right)$ electrolyte of $p \mathrm{H} \sim 6.2$ at room temperature, it is shown that the photo-oxidation can proceed at a rate that is one order of magnitude slower on the nonpolar plane of $\{1 \overline{1} 00\}_{\mathrm{GaN}}$ than on the polar plane of $\{000 \overline{1}\}_{\mathrm{GaN}}$ due to the reduced surface field action. Gallium nitride microcavities bounded by optically smooth $\{1 \overline{1} 00\}$ and $\{1 \overline{1} 03\}$ facets can thus be preferentially formed on the $c$-plane sapphire substrate after dissolving the oxide layer. The optical properties of these GaN hexagonal cavities reveal characteristic peaks of whispering gallery modes in resonance with the $\mathrm{GaN}$ band edge emission spectrum. A typical cavity $Q$ factor of $10^{3}$ is observed in these GaN microcavities due to a reduced optical scattering loss in the wet chemical reaction process. () 2005 American Institute of Physics. [DOI: 10.1063/1.2103423]
\end{abstract}

Semiconductor microcavities are known to play an important role in enhancing the functionality of modern optoelectronic devices. The underlying physics is that a designated cavity can alter the distribution function of the local optical modes into which the electronic transitions can radiate. ${ }^{1}$ One therefore can manipulate the optical process by resorting to a material system upon which the electronic states and optical modes are confined. ${ }^{2}$ This can lead to interesting phenomena such as Purcell enhancement of the spontaneous emission in the weak coupling regime, and entanglement of the cavity mode with exciton when they are brought into resonance in the strong coupling regime. ${ }^{3}$ When the interaction between the optically active medium and the cavity provides a feedback to the generation or switching of coherent light, one can further realize novel light sources such as low-threshold microdisk laser, ${ }^{4}$ or photonic circuitry components ${ }^{5}$ such as tunable add-drop filter/switch, and modulator. A typical structure of semiconductor microcavity would consist of a Fabry-Pérot resonator with pairs of Bragg reflectors in the $z$ direction, whereas in the transverse plane deeply etched holes, pillars, or gratings are sought to partially confine the optical modes. ${ }^{6}$ Two- or three-dimensional optical mode confinement can further be realized in the socalled whispering gallery (WG) cavity in which photons can skim along the curved circumference and encounter repeated total internal reflections. ${ }^{7}$

Gallium nitride $(\mathrm{GaN})$, on the other, is an emerging wide band gap semiconductor whose superior optical and electronic properties have made it a material of choice for short wavelength laser diode (LD) and light emitting diode (LED), ${ }^{8}$ microphotonics devices, ${ }^{9}$ and high-power microwave electronics. ${ }^{10}$ Recent study on GaN microcavities has revealed rich linear and nonlinear characteristics that will not be possible in an otherwise unpatterned, planar structure. For example, enhanced second-harmonic generation can be observed on GaN with two-dimensional distribution of etched holes ${ }^{11}$ and on one-dimensional structure of etched grating. ${ }^{12}$ In addition, large size of GaN microdisk (with diameter

\footnotetext{
${ }^{a)}$ Electronic mail: peng@cc.ee.ntu.edu.tw
}

$>250 \mu \mathrm{m})$ has recently been shown to support stimulated emission and lasing due to the resonance with the cavity WG modes. ${ }^{13,14}$ These observations clarify the importance of including GaN microcavity structures to enhance the device functionality. The chemical stability of GaN, however, limits the cavities to be formed by the dry etching techniques such as reactive ion etching or inductively coupled plasma. However, it is known that a practice of plasma etching can cause surface damage and preferential loss of the nitrogen element in GaN. ${ }^{15}$ Irregular periphery, rough sidewalls, and nonstoichiometric defects thus left on the surface of GaN microcavities can introduce significant scattering loss as one reduces the device dimension. ${ }^{16,17}$ These are the concerns that lead to the $Q$-spoiling effect in the small size GaN cavity, ${ }^{13}$ and loss of grating reflectivity due to the etching-induced material disorder. ${ }^{18}$ It therefore would be desirable if alternative methods that are immune to such problems could be developed.

It has been know that photoelectrochemical (PEC) reaction on $\mathrm{GaN},{ }^{19}$ upon which the photogenerated hot carriers assist the oxidative dissolution of nitride when immersed in an appropriate electrolyte, can provide a smoothly etched surface as well as to preserve the surface stoichiometry. ${ }^{20}$ However, the etch anisotropy associated with the arrangement of electrodes and diffusion of reactants restrict the availability of GaN crystallographic planes to be exposed by the photoenhanced wet etching method. ${ }^{21}$ It has recently been established that unless immersed in molten potassium hydroxide $(\mathrm{KOH})$ or hot phosphoric acid $\left(\mathrm{H}_{3} \mathrm{PO}_{4}\right)$, no etching planes other than $\{10 \overline{1} 0\}$ and $\{10 \overline{1} \overline{1}\}$, or $\{10 \overline{1} \overline{2}\}$ and $\{10 \overline{1} 3\}$, respectively, can be revealed by the etching solutions. ${ }^{22}$ In this work, we demonstrate a novel approach, viz. photoenhanced wet oxidation followed by dissolution, to form $\mathrm{GaN}$ microcavities. By taking advantage of the oxidation rate difference in the polar and nonpolar GaN crystallographic planes, microcavities bounded by smooth and vertical facets of $\{1 \overline{1} 00\}$, or wedged facets of $\{1 \overline{1} 03\}$ can be preferentially formed on the (0001) basal plane of GaN. 


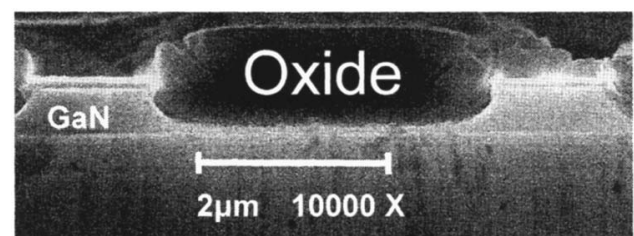

FIG. 1. Cross-sectional SEM micrograph showing a trapezoidal shape of oxide front after $2 \mathrm{~h}$ of PEC oxidation. The total oxide thickness is $1.1 \mu \mathrm{m}$.

The GaN samples used in this study were grown on $c$-plane sapphire substrates by using a commercial metalorganic chemical vapor deposition reactor at Tekcore Corporation (www.tekcore.com.tw). Our device fabrication began with a standard photolithography and metal lift-off process to have a thin $(\sim 100 \mathrm{~nm})$ layer of titanium (Ti) stripe or hexagon patterns with edges aligned parallel to the $\langle 11 \overline{2} 0\rangle_{\mathrm{GaN}}$ direction. The photoenhanced wet oxidation was proceeded in a galvanic photocell consisting of a Ti-patterned GaN working electrode, a platinum $(\mathrm{Pt})$ counter electrode, an UV mercury light source of $254 \mathrm{~nm} .{ }^{23}$ To stabilize the $\mathrm{H}^{+} / \mathrm{H}_{2}$ and $\mathrm{OH}^{-} / \mathrm{O}_{2}$ redox levels with respect to the $\mathrm{GaN}$ surface Fermi level during the photochemistry reaction, a buffered acetic acid $\left(\mathrm{CH}_{3} \mathrm{COOH}\right)$ electrolyte of $p \mathrm{H}$ value $\sim 6.2$ was prepared by adding a small amount of ammonium acetate $\left(\mathrm{CH}_{3} \mathrm{COONH}_{4}\right)$ in the solution. Details on the galvanic cell design for the PEC reaction of $\mathrm{GaN}$ can be found in our recent publications. ${ }^{24,25}$

Illustrated in Fig. 1 is a cross-sectional view of the SEM micrograph revealing the oxide profile after $2 \mathrm{~h}$ of PEC reaction. Note the $1.3 \mu \mathrm{m}$ wide of $\mathrm{Ti}$ stripe, parallel to $[11 \overline{2} 0]_{\mathrm{GaN}}$, was slightly deformed due to the stress force exerted by the laterally overgrown oxide. The oxide front appears to have a trapezoidal profile with a $\sim 2: 1$ ratio of thickness distribution protruding above and below the 3.5$\mu \mathrm{m}$-wide opening of the original $\mathrm{GaN}$ surface. The total thickness of the dehydrated oxide is $\sim 1.1 \mu \mathrm{m}$. These observations reveal a process of volume expansion during the transformation of GaN into gallium oxide $\left(\mathrm{Ga}_{2} \mathrm{O}_{3}\right)$. We denote a slower sidewise oxidation rate along $[1 \overline{1} 00]_{\mathrm{GaN}}$ that is about $\sim 1 / 10$ of that $(3 \mathrm{~nm} / \mathrm{min})$ along $[000 \overline{1}]_{\mathrm{GaN}}$. It resembles the case of lateral overgrowth of $\mathrm{GaN}$ upon which the growth rate along $[0001]_{\mathrm{GaN}}$ is faster than that along $[1 \overline{1} 00]_{\mathrm{GaN}} \cdot{ }^{26}$ Here, however, the photoenhanced electrochemical reaction (i.e., oxidation) not only depends on the surface energy but also on the surface field of a given GaN crystallographic plane. For example, surface band bending of $\mathrm{GaN}$ caused by the surface states and discontinuity of spontaneous polarization ${ }^{27}$ can induce an internal field along $[000 \overline{1}]$ and thus enhance the photo-oxidation process thereupon, whereas in the nonpolar plane of $(1 \overline{1} 00)_{\mathrm{GaN}}$ and $(11 \overline{2} 0)_{\mathrm{GaN}}$ such field action is minimized. ${ }^{28}$

A subtle point that can be inferred from Fig. 1 is the appearance of vertical $\{1 \overline{1} 00\}_{\mathrm{GaN}}$ planes that were found recessed from the electrode edges. This observation indicates that lateral oxidation may take place underneath the electrode due to the local diffusion of reactants in the galvanic cell. It differs from a common observation that the PEC reaction of GaN such as etching would cease at the electrode edges. ${ }^{21}$ Further investigation was made by resorting to a second set of experiments in which a $\sim 1: 1$ ratio of metal Downloaded 09 Feb 2009 to 140.112.113.225. Redistribution subject

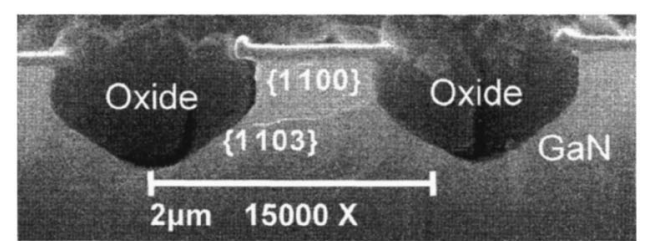

FIG. 2. Cross-sectional SEM micrograph showing a pentagon shape of oxide front after a repeated oxidation and etching process.

grating: GaN opening of $1.2 \mu \mathrm{m}$ width were used to photooxidize a $4-\mu \mathrm{m}$-thick GaN sample. Here we adapt a repeated oxidation-etching scheme to allow a deep photo-oxidation of GaN. The resultant oxide profile is illustrated in Fig. 2, where a pentagon shape of oxide front bounded by vertical facets of $\{1 \overline{1} 00\}_{\mathrm{GaN}}$ and high-index planes of $\{1 \overline{1} 03\}_{\mathrm{GaN}}$ inclined at an angle $\sim 60^{\circ}$ to the substrate normal are clearly resolved.

Since the oxidized GaN layer can be dissolved in dilute acid or base solutions, a combination of the repeated photoenhanced wet oxidation and dissolution (etching) can thus be a promising route for the realization of $\mathrm{GaN}$ microcavities. Illustrated in Fig. 3 are the SEM micrographs showing the top view of $\mathrm{GaN}$ hexagonal microcavities formed by dissolving a $1-\mu$ m-thick oxidized $\mathrm{GaN}$ structure in (a) dilute $\mathrm{CH}_{3} \mathrm{COOH}$ solution and (b) molten $\mathrm{KOH}$ at $180^{\circ} \mathrm{C}$ for $1 \mathrm{~min}$, respectively. The magnified cross-sectional SEM micrograph in the inset of Fig. 3(a) indicates a smooth sidewall can be retained by this proposed method. Except for a partial removal of the Ti mask by a short time immersion in molten $\mathrm{KOH}$, no apparent difference can be found on the sidewall smoothness with respect to the choice of oxide dissolving solutions used in Figs. 3(a) and 3(b). These observations elucidate a principle to retain smooth $\mathrm{GaN}$ facets by slowly photo-oxidizing the nonpolar $\mathrm{GaN}$ planes at a rate much less than $1 \mathrm{~nm} / \mathrm{min}$. In comparison, for the polar $\{0001\}_{\mathrm{GaN}}$ plane where the photoenhanced oxidation rate can proceed at a rate of $1 \mathrm{~nm} / \mathrm{min}$ or higher, a wavy oxidized surface can be observed due to a localized, nonuniform distribution of the photoexcited carriers and reactants. ${ }^{20}$

Note the appearance of slightly rounded hexagons is due to an imperfect lithographic process as can be seen to follow

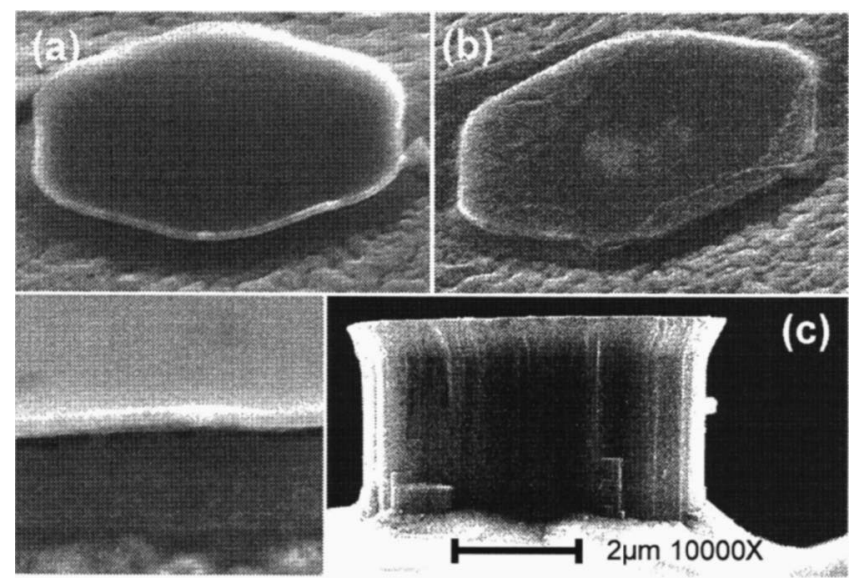

FIG. 3. Top view of SEM micrograph showing GaN hexagonal cavities after dissolving the PEC-grown oxide in (a) $\mathrm{CH}_{3} \mathrm{COOH}$ and (b) molten $\mathrm{KOH}$. Inset: magnified cross-sectional SEM micrograph revealing an optically smooth sidewall in (a). (c) Cross-sectional SEM micrograph of a typical hexagonal GaN cavity to be used in the microphotoluminescence study. The hexagon side length is $2 \mu \mathrm{m}$.

to AIP license or copyright; see http://apl.aip.org/apl/copyright.jsp 


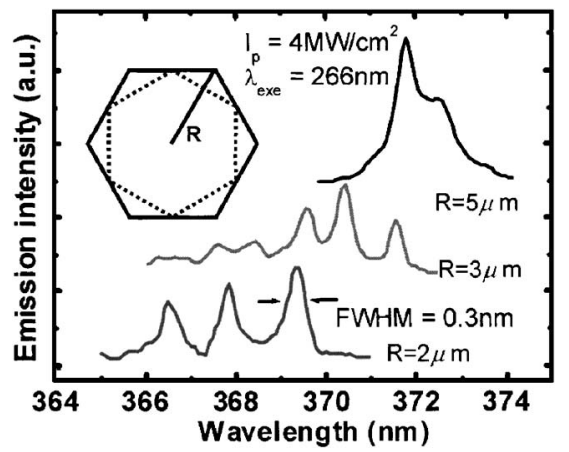

FIG. 4. Cavity size dependent emission spectra of GaN hexagons with radius $R=5,3$, and $2 \mu \mathrm{m}$ measured at an intensity $I \sim 4 \mathrm{MW} / \mathrm{cm}^{2}$ of $266 \mathrm{~nm}$ YAG laser. The spectra have been vertically shifted for comparison. Inset: optical path (dashed line) showing repeated total internal reflections in the GaN hexagonal cavity.

the curved edge of the top Ti mask in the inset of Fig. 3(a). This feature, however, should not affect the basic optical properties of the GaN WG modes which seek repeated total internal reflections from each side of the hexagon as shown in the inset of Fig. $4 .{ }^{29}$ We therefore applied a microphotoluminescence technique to examine the cavity size effect on the emission properties of the WG modes. To avoid background luminescence from the unoxidized GaN, the hexagonal cavities were fabricated to have a height reaching the thickness of the epilayer. Illustrated in Fig. 3(c) is one such hexagon of $2 \mu \mathrm{m}$ radius, showing a smooth, vertical sidewall and inclined $\{1 \overline{1} 03\}_{\mathrm{GaN}}$ facets near the cavity pedestal. The microphotoluminescence experiments were performed by using a $266 \mathrm{~nm} \mathrm{Nd:yttrium-aluminum-garnet} \mathrm{(YAG)} \mathrm{pulse} \mathrm{la-}$ ser of $500 \mathrm{ps}$ width and $20 \mathrm{kHz}$ repetition rate (JDS Uniphase, CA). The samples were backilluminated with a white light LED such that individual hexagon can be imaged and pumped via a telescope system.

Illustrated in Fig. 4 are the cavity size dependent luminescence spectra of GaN hexagons, revealing the characteristic peaks of WG modes in resonance with the GaN emission spectrum. Note a mode spacing of $0.74,1.1$, and $1.5 \mathrm{~nm}$, respectively, are clearly discernible with a reduced hexagon radius of $R=2,3$, and $5 \mu \mathrm{m}$ at a constant pump intensity $I_{p} \sim 4 \mathrm{MW} / \mathrm{cm}^{2}$. These data agree well with a theoretical analysis by enforcing an optical path (shown in the inset of Fig. 4) to have a total phase shift equal to an integer multiple of $2 \pi$ and thus to ensure total internal reflections inside the GaN microcavities. ${ }^{29,30}$ Here the wavelength shift of the GaN band edge emission from a bulk value of $365 \mathrm{~nm}$ to a long wavelength side is caused by the carrier-induced band gap renormalization effect. ${ }^{13}$ The cold (empty) cavity $Q$ factor can be evaluated by measuring the spectral width $\Delta \lambda$ at a pump intensity below the threshold and $Q=\lambda / \Delta \lambda$, where $\lambda$ is the emission peak wavelength of the dominant WG mode. $^{31}$ A typical value of $Q \sim 10^{3}$ can thus be inferred from our $2 \mu \mathrm{m}$ size GaN hexagon cavity, proving an immunity capability to the $Q$-spoiling effect with reduced device size. Note this cavity $Q$ factor is comparable to that in the stateof-the-art $\mathrm{GaN}$ wires prepared by the growth method. ${ }^{32}$ These observations support our proposal of using the photochemical oxidation as a promising route for the realization of high- $Q$ GaN cavity that can have profound applications in the photonic devices.

This research was supported by the National Science Council, Grant Nos. NSC 92-2215-E-002-027, 2120-M-001005, and NSC 94-2120-M-009-007.

${ }^{1}$ M. Bayer, A. Forchel, Th. L. Reinecke, P. A. Knipp, and S. Rudin, Phys. Status Solidi A 191, 3 (2002).

${ }^{2}$ Confined Electrons and Photons NATO ASI series B: Physics Vol. 340, edited by E. Burstein and C. Weisbuch (Plenum, New York, 1995).

${ }^{3}$ For a recent review, please see K. J. Vahala, Nature (London) 424, 839 (2003); Optical Processes in Microcavities, edited by R. K. Chang and A. J. Campillo (World Scientific, Singapore, 1996).

${ }^{4}$ For a recent review, please see T. Baba, IEEE J. Sel. Top. Quantum Electron. 3, 808 (1997)

${ }^{5}$ K. Djordjev, S. J. Choi, S. J. Choi, and P. D. Dapkus, J. Lightwave Technol. 20, 105 (2002).

${ }^{6}$ N. Yokouchi, A. J. Danner, and K. D. Choquette, IEEE J. Sel. Top. Quantum Electron. 10, 1439 (2003).

${ }^{7}$ S. L. McCall, A. F. J. Levi, R. E. Slusher, S. J. Pearton, and R. A. Logan, Appl. Phys. Lett. 60, 289 (1992).

${ }^{8}$ S. Nakamura and G. Fasol, The Blue Laser Diode (Springer, Berlin, 1997).

${ }^{9}$ H. X. Jiang and J. Y. Lin, Crit. Rev. Solid State Mater. Sci. 28, 131 (2003).

${ }^{10}$ M. A. Khan, G. Simin, J. Yang, J. Zhang, A. Koudymov, M. S. Shur, R. Gaska, X. Hu, and A. Tarakji, IEEE Trans. Microwave Theory Tech. 51, 624 (2003).

${ }^{11}$ G. Vecchi, J. Torres, D. Coquillat, M. Le V. d'Yerville, and A. M. Malvezzi, Appl. Phys. Lett. 84, 1245 (2004).

${ }^{12}$ J. Torres, D. Coquillat, R. Legros, J. P. Lascaray, F. Teppe, D. Scalbert, D. Peyrade, Y. Chen, O. Briot, M. Le V. d'Yerville, E. Cassagne, and J. P. Albert, Phys. Rev. B 69, 085105 (2004).

${ }^{13}$ S. Chang, N. B. Rex, R. K. Chang, G. Chong, and L. J. Guido, Appl. Phys. Lett. 75, 166 (1999).

${ }^{14}$ M. Kneissl, M. Teepe, N. Miyashita, N. M. Johnson, G. D. Chern, and R. K. Chang, Appl. Phys. Lett. 84, 2485 (2004).

${ }^{15}$ K. J. Choi, H. W. Jang, and J.-L. Lee, Appl. Phys. Lett. 82, 1233 (2003).

${ }^{16}$ K. C. Zeng, L. Dai, J. Y. Lin, and H. X. Jiang, Appl. Phys. Lett. 75, 2563 (1999).

${ }^{17}$ E. D. Haberer, R. Sharma, C. Meier, A. R. Stonas, S. Nakamura, S. P. DenBaars, and E. L. Hu, Appl. Phys. Lett. 85, 5179 (2004).

${ }^{18}$ C. Marinelli, M. Bordovsky, L. J. Sargent, M. Gioannini, J. M. Rorison, R. V. Penty, I. H. White, P. J. Heard, M. Benyoucef, M. Kuball, G. Hasnain, T. Takeuchi, and R. P. Schneider, Appl. Phys. Lett. 79, 4076 (2001).

${ }^{19}$ M. S. Minsky, M. White, and E. L. Hu, Appl. Phys. Lett. 68, 1531 (1996).

${ }^{20}$ C. Youtsey, I. Adesida, L. T. Romano, and G. Bulman, Appl. Phys. Lett. 72, 560 (1998).

${ }^{21}$ C. Youtsey, I. Adesida, and G. Bulman, Appl. Phys. Lett. 71, 2151 (1997).

${ }^{22}$ D. A. Stocker, E. F. Schubert, and J. M. Redwing, Appl. Phys. Lett. 73, 2654 (1998).

${ }^{23}$ L.-H. Peng, C.-H. Liao, Y.-C. Hsu, C.-S. Jong, C.-N. Huang, J.-K. Ho, C.-C. Chiu, and C.-Y. Chen, Appl. Phys. Lett. 76, 511 (2000).

${ }^{24}$ L.-H. Peng, C.-W. Chuang, J.-K. Ho, C.-N. Huang, and C.-Y. Chen, Appl. Phys. Lett. 72, 939 (1998).

${ }^{25}$ L.-H. Peng, C.-W. Chuang, Y.-C. Hsu, J.-K. Ho, C.-N. Huang, and C.-Y. Chen, IEEE J. Sel. Top. Quantum Electron. 4, 564 (1998).

${ }^{26} \mathrm{H}$. Matsushima, M. Yamaguchi, K. Hiramatsu, and N. Sawaki, J. Cryst. Growth 189/190, 78 (1998)

${ }^{27}$ L.-H. Peng, C.-W. Shih, C.-M. Lai, C.-C. Chuo, and J.-I. Chyi, Appl. Phys. Lett. 82, 4268 (2003).

${ }^{28}$ T. Takeuchi, H. Amano, and I. Akasaki, Jpn. J. Appl. Phys., Part 1 39, 413 (2000).

${ }^{29}$ T. Nobis, E. M. Kaidashev, A. Rahm, M. Lorenz, and M. Grundmann, Phys. Rev. Lett. 93, 103903 (2004).

${ }^{30}$ S. Bidnyk, B. D. Little, Y. H. Cho, J. Krasinski, J. J. Song, W. Yang, and S. A. McPherson, Appl. Phys. Lett. 73, 2242 (1998).

${ }^{31}$ R. E. Slusher, A. F. J. Levi, U. Mohideen, S. L. McCall, S. J. Pearton, and R. A. Logan, Appl. Phys. Lett. 63, 1310 (1993).

${ }^{32}$ J. C. Johnson, H.-J. Choi, K. P. Knutsen, R. D. Schaller, P. Yang, and R. J. Saykally, Nat. Mater. 1, 106 (2002). 